\title{
IMPACTO DO USO IRRACIONAL DE MEDICAMENTOS PEDIÁTRICOS E OS DESAFIOS PARA A PROFISSÃO FARMACÊUTICA
}

\author{
A.S. FERREIRA², M.J.S. LIMA", S.A.M.S. ALVES', A.C. SILVA², R.M.F. SILVA ${ }^{1}$, P.J. \\ ROLIM NETO ${ }^{1}$ \\ ${ }^{1}$ Universidade Federal de Pernambuco, Centro de Ciências da Saúde, Departamento de \\ Ciências Farmacêutica \\ ${ }^{2}$ Centro Universitário Maurício de Nassau, Departamento de Ciências Farmacêutica \\ E-mail para contato: joanellys.lima@hotmail.com
}

\begin{abstract}
RESUMO - A assistência farmacêutica emergiu como garantia do acesso, uso apropriado de medicamentos e racionalização dos custos. Nesse contexto, surge à prática da automedicação que pode ser adequada quando feita de maneira correta, porém há o risco de ocasionar danos à saúde, sendo um fato expressivo ao se tratar de crianças. Assim o objetivo foi avaliar a importância da assistência farmacêutica na pediatria. Baseou-se em um estudo observacional com corte transversal de 2010 à 2017. Observa-se que há um grande número de praticantes da automedicação em crianças, sendo a mãe responsável por 95\% dos casos. O maior motivo relatado por essas pessoas é a praticidade. Conclui-se que a atenção à saúde da população pediátrica revela-se cercada por muitos desafios na área de Assistência Farmacêutica. Sendo essencial a promoção de políticas públicas que transformem o quadro de dificuldades assistenciais e tragam avanços para a terapêutica pediátrica.
\end{abstract}

Palavras-chave: Uso racional de medicamento. Automedicação. Crianças.

\begin{abstract}
Pharmaceutical assistance emerged as a guarantee of access, appropriate use of medicines and rationalization of costs. In this context, it appears to the practice of self-medication that can be adequate when done correctly, but there is a risk of causing damage to health, being an expressive fact when dealing with children. Thus the objective was to evaluate the importance of pharmaceutical assistance in pediatrics. It was based on an observational cross-sectional study from 2010 to 2017. It is observed that there are a large number of practitioners of self-medication in children, with the mother responsible for 95\% of the cases. The biggest motive reported by these people is the practicality. It is concluded that the health care of the pediatric population is surrounded by many challenges in the area of Pharmaceutical Care. It is essential to promote public policies that transform the framework of care difficulties and bring advances to pediatric therapy.
\end{abstract}

Key words: Rational use of medicines. Self-medication. Children. 


\section{INTRODUÇÃO}

Com a descentralização político-administrativa do SUS e o surgimento da Política Nacional de Medicamentos, emergiu a necessidade da assistência farmacêutica para garantir acesso e uso apropriado de medicamentos de qualidade, além da racionalização dos custos (Silva et. al., 2016).

Dentro do âmbito da assistência farmacêutica, a automedicação é uma prática que vem sendo bastante observada e discutida, apresentando-se como um dos problemas de maior complexidade da saúde pública. Segundo a Organização Mundial de Saúde (OMS) metade dos medicamentos são prescritos ou vendidos de maneira inadequada e desses cerca de 50\% são consumidos de forma inapropriada (Goulart et. al., 2012).

Sabe-se que os principais usuários dos serviços de saúde nos países em desenvolvimento são as crianças e o seu padrão de enfermidades se refletem no consumo de medicamentos, este por sua vez, pode ser excessivo, uma prática muito difundida induzida pela mídia e realizada sem a indicação e a prescrição médica (Cruz et. al, 2014). Este trabalho tem como objetivo avaliar a importância da assistência farmacêutica na pediatria.

\section{MATERIAIS E MÉTODOS}

Baseou-se em um estudo observacional com corte transversal de 2010 à 2017, utilizando legislações e periódicos obtidos das bases de dados: Scielo, ScienceDirect e Bireme.

\section{RESULTADOS E DISCUSSÃO}

Estudos realizados no tocante da automedicação na população pediátrica mostram quem são os principais responsáveis, a faixa etária das crianças, as justificativas e sintomas, os medicamentos mais utilizados e as principais fontes para consegui-los (Beckhauser et. al., 2010; Filho \& Pereira Junior, Prolungatti et. al., 2014; Goulart et. al., 2012; Cruz et. al, 2014).

Quadro 1 - Situação da automedicação na população pediátrica.

\begin{tabular}{|c|c|c|c|c|}
\hline $\begin{array}{l}\text { Principais } \\
\text { responsáveis }\end{array}$ & $\begin{array}{l}\text { Faixa } \\
\text { etária das } \\
\text { crianças }\end{array}$ & Justificativas utilizadas & $\begin{array}{l}\text { Principais } \\
\text { medicamentos }\end{array}$ & $\begin{array}{l}\text { Principais } \\
\text { fontes }\end{array}$ \\
\hline $\begin{array}{l}\text { Mães } \\
\text { Avós }\end{array}$ & $0-14$ anos & $\begin{array}{l}\text { Já ter utilizado antes; } \\
\text { Já possuir o medicamento em casa; } \\
\text { Aproveitamento da receita de } \\
\text { irmãos mais velhos; } \\
\text { Médico ter receitado anteriormente; } \\
\text { Influencia da televisão; } \\
\text { Associação de um medicamento a } \\
\text { um sintoma. Ex: Dipirona para } \\
\text { febre o mais comum; } \\
\text { Sintomas comuns como febre, } \\
\text { asma, cólicas, resfriados, diarreia. }\end{array}$ & $\begin{array}{l}\text { Dipirona; } \\
\text { Paracetamol; } \\
\text { Xaropes } \\
\text { expectorantes; } \\
\text { Amoxicilina; } \\
\text { Simeticona; } \\
\text { Metoclopramida. }\end{array}$ & $\begin{array}{l}\text { Farmácias } \\
\text { comerciais; } \\
\text { Unidades de } \\
\text { saúde. }\end{array}$ \\
\hline
\end{tabular}

Fonte: Beckhauser et. al., 2010 Filho \& Pereira Junior, Prolungatti et. al., 2014; Goulart et. al., 2012; Cruz et. al, 2014.

A partir de estudos pode-se observar que a prática da automedicação em crianças é algo muito comum e por isso preocupante, pois a dose, administração e duração do tratamento podem ser inadequados. Dessa forma, pode ocasionar danos a saúde, como 
mascaramento de sintomas, interações medicamentosas e intoxicações, onde compromete diretamente a eficácia e segurança do tratamento. Fato expressivo quando ao se tratar de crianças (Beckhauser et. al., 2010 Filho et. al., 2014; Goulart et. al., 2012; Cruz et. al, 2014). Onde, grande parte dos estudos apontam medicamentos e produtos químicos como um dos principais fatores de acidentes tóxicos que levam à internação e morte de crianças (Martins, 2006).

Visando a redução desta prática foi publicada em 2015 a Política Nacional de Atenção Integral à Saúde da Criança (Pnaisc), que estabelece, entre outras ações: "atenção integral a crianças com agravos prevalentes na infância e com doenças crônicas" articulada a ações de Assistência Farmacêutica (Brasil, 2015).

Nesse contexto, destaca-se a importância do papel do farmacêutico na assistência farmacêutica, orientando quanto ao Uso Racional de Medicamentos (URM), que envolve o recebimento dos medicamentos apropriados pelo paciente, voltados para suas necessidades clínicas, em doses adequadas, pelo período necessário e a um custo razoável (Wannmacher, L., 2012), onde abrange a prescrição, dispensação e uso propriamente dito, sendo imprescindível que os profissionais farmacêuticos disponibilizem seus conhecimentos, durante a orientação aos pacientes, de forma adequada e segura, reforçando o consumo consciente dos medicamentos, bem como o elevado potencial de gravidade da automedicação.

Nesse sentido o Ministério da Saúde em 2017, aponta as seguintes recomendações e estratégias.

Quadro 2 - Recomendações e estratégias para o "Eixo Viii - Formação e educação para o uso racional de medicamentos"

\begin{tabular}{|c|c|c|}
\hline $\begin{array}{l}\text { Promover atividades sobre o } \\
\text { URM em a Pediatria, } \\
\text { direcionadas aos cidadãos, } \\
\text { especialmente pais, cuidadores } \\
\text { e educadores. }\end{array}$ & \begin{tabular}{lcc} 
Estimular & e & promover a \\
presença de & farmacêutico \\
clínico com & \multicolumn{2}{c}{ capacitação } \\
específica na & rotina das \\
unidades de assistência à saúde \\
da população pediátrica.
\end{tabular} & $\begin{array}{l}\text { Formar/capacitar profissionais } \\
\text { da saúde, especialmente } \\
\text { dentistas, enfermeiros, } \\
\text { farmacêuticos e médicos, sobre } \\
\text { o URM em RN, lactentes, } \\
\text { crianças e adolescentes. }\end{array}$ \\
\hline $\begin{array}{l}\text { Identificar e promover parcerias } \\
\text { entre representantes dos } \\
\text { trabalhadores e gestores. }\end{array}$ & \multirow[t]{2}{*}{$\begin{array}{lcc}\text { Promover a } & \text { capacitação } & \text { de } \\
\text { farmacêuticos } & \text { clínicos } & \mathrm{em} \\
\text { Pediatria. } & & \\
\end{array}$} & $\begin{array}{l}\text { Incluir e aprimorar os conteúdos } \\
\text { de promoção do URM em } \\
\text { Pediatria no ensino. }\end{array}$ \\
\hline $\begin{array}{l}\text { "URM em Pediatria" } \\
\text { atividades da educação. }\end{array}$ & & $\begin{array}{l}\text { Promover eventos direcionados à } \\
\text { temática do URM em Pediatria. }\end{array}$ \\
\hline \multirow{3}{*}{$\begin{array}{l}\text { Utilização das práticas } \\
\text { integrativas e complementares } \\
\text { para redução do uso abusivo de } \\
\text { medicamentos em crianças. }\end{array}$} & \multirow[t]{3}{*}{$\begin{array}{l}\text { Inserir o farmacêutico na equipe } \\
\text { multiprofissional de saúde. }\end{array}$} & $\begin{array}{llr}\text { Promover } & \text { parcerias } & \text { para } \\
\text { elaboração de cursos } & \text { de } \\
\text { capacitação sobre o URM. } & \end{array}$ \\
\hline & & $\begin{array}{l}\text { Identificar atores para } \\
\text { disseminação de conhecimentos. }\end{array}$ \\
\hline & & $\begin{array}{l}\text { Promover a notificação } \mathrm{de} \\
\text { eventos adversos. }\end{array}$ \\
\hline
\end{tabular}

Fonte: BRASIL, Ministério da saúde. 2017.

Como foi possível observar a cartilha ao tratar da Assistência Farmacêutica em

Pediatria no Brasil, apresenta valiosas contribuições para a saúde pública e melhorias nas condições terapêuticas relacionadas ao uso racional de medicamentos, também servindo de guia para a população.

\section{CONCLUSÃO}

A atenção à saúde da população pediátrica revela-se cercada por muitos desafios na área de Assistência Farmacêutica. Contudo, a automedicação ainda é um problema que 
pode causar danos sérios a saúde, sendo essencial a promoção de políticas públicas, que transformem o quadro de dificuldades assistenciais, produzindo conhecimentos, possibilitando intervenções e implementações estratégicas, sobretudo de cunho educativo, objetivando a conscientização dos pais e/ou responsáveis, a fim de garantir segurança terapêutica para as crianças.

\section{REFERENCIAS}

BECKHAUSER, G. C. et al. Utilização de medicamentos na pediatria: A prática de automedicação em crianças por seus responsáveis. Rev. paul. Pediatr., 2010.

BRASIL. Ministério da Saúde. Portaria $n^{\circ} 1.130$, de 5 de agosto de 2015. Institui a Política Nacional de Atenção Integral à Saúde da Criança (PNAISC) no âmbito do Sistema Único de Saúde (SUS). Diário Oficial da União, Brasília, 2015.

BRASIL. Ministério da saúde. Assistência Farmacêutica em Pediatria no Brasil: recomendações e estratégias para a ampliação da oferta, do acesso e do Uso racional de Medicamentos em crianças. Brasília, 2017.

CRUZ, M. J. B. et al. Medication use among children 0-14 years old: Population baseline study. J. Pediatr., 2014.

FILHO, P. C. P. T.; PEREIRA JÚNIOR, A. C. Automedicação em crianças de zero a cinco anos: fármacos administrados, conhecimentos, motivos e justificativas. Esc Anna Nery, 2013.

GOULART, I. DA C. et al. Automedicação em menores de cinco anos em municípios do Pará e Piauí: prevalência e fatores associados. Rev. Bras. Saude Mater. Infant., 2012.

MARTINS, C. B. G. acidentes na infância e adolescência: uma revisão bibliográfica. Rev. Bras. Enferm., Brasília, 2006.

PROLUNGATTI, C. N. et. al. Use of analgesic and anti-inflammatory drugs before assistance in a children's first aid unit. Revista Dor, 2014.

SILVA, R. M. et. al. Assistência farmacêutica no município do Rio de Janeiro, Brasil: evolução em aspectos selecionados de 2008 a 2014. Ciênc. Saúde Coletiva, 2016.

WANNMACHER, L. Condutas baseadas em evidências sobre medicamentos utilizados em atenção primária à saúde. BRASIL. Ministério da Saúde. Uso Racional de Medicamentos: temas selecionados. Brasília: Ministério da Saúde, 2012. 